\title{
Residential Factors Affecting Quality of Life of People with Disabilities
}

\author{
Eun Hye Jung ${ }^{1 *}$ and Yeong Hun Yeo ${ }^{2}$ \\ ${ }^{1 *} \mathrm{Ph}$. D candidate of Social Welfare, Jeonbuk National University, Korea \\ ${ }^{2}$ Associate professor of Social Welfare, Jeonbuk National University, Korea \\ I*dideun@gmail.com, ${ }^{2} y$ hyeo2@gmail.com
}

\begin{abstract}
This study examined factors affecting the quality of life of people with disabilities considering regional levels, especially reviewing the current situation by classifying types of residence into single households and two-or-more-persons households and comparing them. At personal level, identified such as senior people with disabilities, degree of disability, disability types, housing conditions, satisfaction with residential environment, burden on housing maintenance, anxiety over rental and public rental. Variables at the Regional level are live in the metropolitan area, the ratio of people with disability to population, the employment rate of disabled people, rental housing price index, financial autonomy rate and social welfare resources. Personal variables were statistically significant, and variables at the regional level were found to increase statistically significant in quality of life, with a loving in non-metropolitan areas, lower rental price index, and a higher financial autonomy rate. Chow test mainly used to identify different influences of variables among groups in comparison with slopes was conducted to see group differences depending on types of household. As a result, two-or-more-persons households experiences lower quality of life in terms of senior people with disabilities and public rental housing.
\end{abstract}

Keywords: People with disabilities, Residential factors, Life satisfaction

\section{Introduction}

The ultimate aim of social welfare policies and services is to improve the quality of life for subjects [1][2]. The quality of life is a very complex concept that covers not only objective external factors such as the physical environment but also feelings such as satisfaction with the environment, autonomy and choice [3]. People with disabilities living in communities are highly vulnerable to poverty due to a number of limitations and they are disadvantaged at maintaining stable residences. Although countries around the world are providing appropriate services for this matter, people with disabilities are still vulnerable to residential environment that is affecting the quality of their lives. Residence is particularly more crucial in helping them in continuing independent lives.

Support for residential environment for the people with disabilities serves as evidence for enhancing the quality of their lives [4]. For those who live alone without proper life support systems, they are involved with risk elements that could lead to poor quality of life [5]. In spite of this problem, in South Korea, an approach to identify elements related with their

Article History:

Received (November 17, 2019), Review Result (December 22, 2019), Accepted (January 29, 2020) 
residence is practically limited. Due to the fact that housing is largely affected by regions, regional factors should also be taken into account. On the basis of such questions, the aim of this study is to analyze factors affecting the quality of life of people with disabilities, together with the characteristics of personal residential environment and regional factors, and further identify the current situation by comparing whether they live alone.

\section{Method}

\subsection{Data collection}

The study statistically analyzed the most recent survey of people with disabilities in South Korea, the 2015 microdata, as secondary data. As of February 2015, the survey was conducted on 8,004 people with disabilities in total, but the study analyzed 2,760 people by selecting only housing leases out of the total surveyed. Data on the 2015 survey on regional factors was downloaded from the national statistics official website (http://kosis.kr/index/index.do) managed by the Korean National Statistical Office, part of which were collected in a way that claims for obtaining information to the Office.

\subsection{Major variables}

The quality of life, a dependent variable of this study, was measured by the perception of the present state of health, the economy, the next door neighbor and the overall life of the people with disabilities. First of all, gender and single households were defined and controlled as variables to see factors affecting the quality of life.

At a personal level, the elderly was defined based on the age of 60, through degree of disability(mild and severe), types of disability(external physical disability, mental disability and internal organ disability), housing conditions including facade, satisfaction with residential environment with an aim to see content with regional environment, burdens on housing maintenance expense anxiety over rental and public rental were found.

At the regional level, live in the metropolitan area, the ratio of registered people with disabilities to population in regions was turned to percentage, consisting of social welfare resources such as the employment rate of people with disabilities, rental housing price index (Jeonse price index), financial autonomy rate and the number of social welfare facilities with a population of 100,000 .

\subsection{Analytical methods}

This study followed a comparison and tests on factors affecting the quality of life of people with disabilities. Firstly, the current situation was reviewed by using descriptive statistics on major variables and multilevel analysis was carried out. The multilevel analysis is conducted when there are hierarchically structured analytical levels. In this regard, a level-2 personal and regional multilevel model was established in this study.

In addition, Chow test [6] mainly used to identify different influences of variables among groups in comparison with slopes was conducted to see group differences depending on types of households (single or not). SPSS 21 and STATA 13.0 were used as statistical packages.

\section{Results}

\subsection{Descriptive statistics on major variables}


Men accounted for 59.2\% than women; two-person households accounted for $63.3 \%$, which was higher than single person households. Senior people with disabilities accounted for $44 \%$, which were not even lower. The percentage of severe disability turned out $56.5 \%$, which was higher than a mild level. Physical disability accounted for $58.4 \%$. The quality of life, a dependent variable, showed 2.24 less than 2.5, a medium point of 4-point scale. Single households showed 2.16 and two-or-more-persons households showed 2.29. This indicates that the two-or-more-persons households enjoy better quality of life and the difference was significant $\left(\mathrm{t}=6.636^{* * *}\right)$. Housing conditions, satisfaction with residential environment and anxiety over rental showed a relatively higher mean than a medium level of index. The percentage of those living in public rental houses accounted for $44.4 \%$. For regional variables, the percentage of those living in the metropolitan areas accounted for $37.3 \%$; the average percentage of people with disabilities to population was $4.94 \%$; the employment rate of people with disabilities was 3.18\%; an average rental housing price index was 95.55 and financial autonomy rate was $49.51 \%$. There were 13 social welfare resources were with a population of 100,000 .

\subsection{Results of multilevel models}

Multilevel analysis was performed by considering personal and regional hierarchical structures with an aim to examine the effects on the quality of life-based on the characteristics of personal disabilities and regional characteristics. Data shows 171 different areas. Grand mean centering was applied in all the variables among all data. A null model was tested to see the difference of the quality of their lives in rental houses in a bid to apply multilevel models. The results of LR-test aiming to test fixed-effects models and difference were significant (total $\chi^{2}=130.73^{* * *}$, single households $\chi^{2}=46.38^{* * *}$, two-or-more-persons households $\left.\chi^{2}=67.17 * * *\right)$ showed that regional wireless models were appropriate. ICC values by dividing regional distributions by totals of two-level distribution, indicated that the entire group was .105, single households was .124 and two-or-more-persons households was .101. The three groups showed $1.05 \%, 12.4 \%$ and $10.1 \%$ explanatory powers, respectively. Among them was "single households" that showed the highest explanatory power of regional variables.

The table below shows personal factors and regional factors on the quality of life of people with disabilities.

Table 1. Results of multilevel models

\begin{tabular}{|c|c|c|c|c|c|}
\hline \multicolumn{4}{|c|}{ Classification } & Coef. & S.E. \\
\hline \multirow{9}{*}{$\begin{array}{l}\text { Fixed } \\
\text { Effect }\end{array}$} & \multicolumn{3}{|c|}{ Constant } & $2.243 * * *$ & .016 \\
\hline & \multirow{2}{*}{ Control } & \multicolumn{2}{|c|}{ Gender (Women) } & .027 & .017 \\
\hline & & \multicolumn{2}{|c|}{ Household type (Two-or-more) } & $-.098 * * *$ & .018 \\
\hline & \multirow{6}{*}{$\begin{array}{l}\text { Level } 1 \\
\text { Personal } \\
\text { level }\end{array}$} & \multicolumn{2}{|c|}{ Senior people with disabilities (Under the age of 60) } & $-.112 * * *$ & .018 \\
\hline & & \multicolumn{2}{|c|}{ Degree of disability (Mild) } & $-.1 * * *$ & .019 \\
\hline & & \multirow{2}{*}{$\begin{array}{c}\text { Types of disability } \\
\text { (External physical } \\
\text { disability) }\end{array}$} & Mental disability & $.085 * *$ & .026 \\
\hline & & & Internal organ disability & $-.061 * *$ & .022 \\
\hline & & \multicolumn{2}{|c|}{ Housing condition } & $.094 * * *$ & .016 \\
\hline & & \multicolumn{2}{|c|}{ Satisfaction with housing environment } & $.122 * * *$ & .022 \\
\hline
\end{tabular}




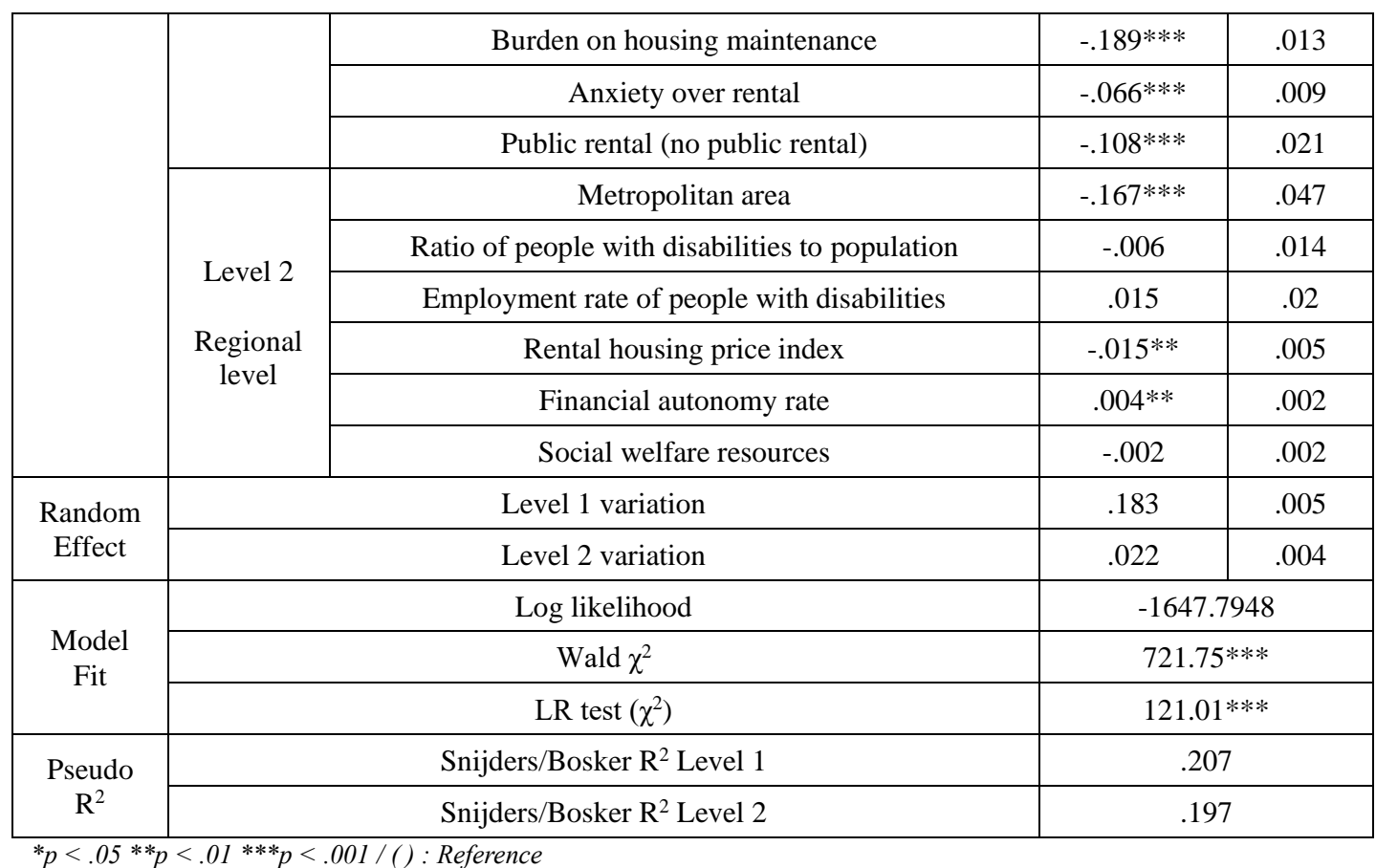

The comparison of null models and multilevel models showed that the later ones showed better goodness of fit. As a result of examining the explanatory power of the models, pseudo $\mathrm{R}^{2}$ value showed that values considering regional level variables showed higher explanatory power than those only considering personal level variables. Two-or-more-person households showed statistically higher quality of life than single households in terms of housing types. Personal variables were statistically significant. Mental disability, fine housing conditions and satisfactory residential environment enhanced the quality of life. It was found that the older the disabled over 60 years old, the more severe the disabled, the greater the burden of housing management and the anxiety caused by rent, and the lower the quality of life for people living in public rental housing. Variables at the regional level were found to increase statistically significantly in quality of life, with a living in non-metropolitan areas, lower rental price index, and a higher financial autonomy rate.

\subsection{Comparison of results of multilevel model depending on single or two-or-more- persons households}

Multilevel models were applied in single households and two-or-more-persons households, respectively, with the aim of identifying different outcomes of factors affecting the quality of their lives depending on single types. Furthermore, Chow test was performed to see the significant difference of both groups. The difference of all the models was statistically significant $\left(\chi^{2}=64.33 * * *\right)$. Observing the different impacts of individual variables, senior people with disabilities $\left(\chi^{2}=10.28 * *\right)$ and anxiety over rental $\left(\chi^{2}=9.21 * *\right)$ showed a stark difference.

\section{Conclusion}


This study examined factors affecting the quality of life of people with disabilities based on regional levels, especially reviewing the current situation by classifying types of residence into single households and two-person households and comparing them.

First, the score of the quality of life of people with disabilities living in rental houses was relatively lower than the mean. Two or more persons households achieved higher scores than single households. As observed in the life of single disabled person is far lower than other people with disabilities living together with their families, single households need to be considered to improve the quality of their lives.

Second, personal disability factors affect the quality of life in case of considering factors such as gender of disabled people, household types and regional society. The more severe level of disabilities, the lower quality of their lives. For those with internal organ disability than physical one experience far lower quality of life. Especially those with internal organ disability, as opposed to external physical disability, are required to received ongoing treatment and pay medical expenses [7]. Therefore, the government needs to actively extend hands to them.

Third, all residential factors among their personal levels affected the quality of life. Personal residence should be considered when it comes to the quality of their lives.

Fourth, regional factors including metropolitan areas, rental housing price index and financial autonomy rate affected the quality of life of disabled people Rental housing price index and metropolitan environment served as a cause for reducing the quality of their lives, while higher financial autonomy rate elevated their lives. The above result suggests that more efforts are need to build better welfare systems for people with disabilities for areas with lower financial autonomy rate, together with improving the quality of their lives in the metropolitan areas. Areas with higher rental housing price index are recommended to establish support systems for those living in rental houses.

Fifth, two-or-more-persons households experiences lower quality of life in terms of senior people with disabilities and public rental housing. Although the differences between the groups were not statistically significant, for types of disability, single people with internal organ disabilities experience poor quality of life than those with external physical disabilities. Those with mental disabilities living in two-or-more-persons households see an increased quality of life. Since those with internal organ disabilities regularly need to receive medical treatment and visit hospitals, maintain a clean environment and avoid potential infection risks [8], they need an appropriate residential environment for easier access to medical services.

In order to improve the quality of life of senior people with disabilities, services for the elderly and services for the disabled must be provided together [9]. Only people with disabilities of two-or-more-persons households living in public rental houses see the lower quality of life. Public rental houses were underrated in earlier studies [10]. Thus, a housing support system tailored to living with families is needed.

This study considered and analyzed regional variables affecting the quality of their lives. It is particularly deemed to have examined variables with different impacts depending on types of residence by comparing single households with two-or-more-persons households.

\section{References}

[1] Brown I., Hatton C., and Emerson E., "Quality of life indicators for individuals with intellectual disabilities: Extending current practice," Intellectual and Developmental Disabilities, vol.51, no.5, pp.316-332, (2013)

[2] Felce D., "Defining and applying the concept of quality of life," Journal of Intellectual Disability Research, vol.41, no.2, pp.126-135, (1997) 
[3] Sines David, Elaine Hogard, and Roger Ellis, "Evaluating quality of life in adults with profound learning difficulties resettled from hospital to supported living in the community," Journal of Intellectual Disabilities, vol.16, no.4, pp. 247-263, (2012)

[4] YoungSuk Kim, "A study on the actual conditions of residential institutions and residential environment support for quality improvement of life of person with disabilities," The Journal of Special Education: Theory and Practice, vol.11, no.2, pp.47-68, (2010)

[5] JuYoung Park and HyeKyung Oh, "A study on influence of the life satisfaction for the elderly women with disabilities living alone," Journal of Disability and Welfare, vol.22, pp.109-128, (2013)

[6] Chow G. C., "Tests of equality between sets of coefficients in two linear regressions.," Econometrica, vol.28, no.3, pp.591-605, (1960)

[7] Jeong Eun Lee, EunKyung Shin, and HyungIk Shin, "Social participation and the effect of environmental factor among persons with disability of internal organs - using WHODAS 2.0," Korean Journal of Social Welfare Research, vol.41, pp.59-88, vol.22, no.4, pp.241-266, (2014)

[8] JinSuk Seo and GwanYong Kim, "A study on affected factors of employment of disabilities of internal organs in Korea," Disability \& Employment, (2012)

[9] Eun Hye Jung and Myeong Sook Yoon, "Social support, depression, self-esteem influences on life satisfaction of disability in aging," Journal of the Korean Gerontological Society, vol.38, no.3, pp.645-666, (2018)

[10] Hee Jung Jun and Soo Jeong Han, "Mental health change among public housing residents: using propensity score matching," The Korea Association for Policy Studies, vol.27, no.4, pp.67-102, (2018) 